\title{
ENTIERROS DE ADULTOS EN URNA EN LA QUEBRADA DE HUMAHUACA. UN CASO DE ESTUDIO DEL PUCARA DE PERCHEL (DTO. TILCARA, JUJUY)
}

\author{
Agustina Scaro $^{1}$ y María Soledad Gheggi ${ }^{2}$ \\ ${ }^{1}$ CONICET. Instituto de Geología y Minería. Universidad Nacional de Jujuy. Av. Bolivia 1661. (4600) \\ San Salvador de Jujuy. agustina.scaro@gmail.com \\ ${ }^{2}$ CONICET. Instituto de Arqueología (FFyL). Universidad de Buenos Aires. 25 de Mayo 217 \\ (C1002ABD) CABA. solelingheggi@yahoo.com.ar
}

Presentado el: 10/11/2010 - Aceptado 15/03/2011

\section{Resumen}

En este trabajo se analiza el hallazgo de un entierro primario de adulto en una urna realizado en el Pucara de Perchel. El Pucara de Perchel tiene $2.921 \mathrm{~m}^{2}$ de superficie y está ubicado en el Angosto de Perchel (dpto. Tilcara). El sitio habría sido ocupado con mayor intensidad durante los períodos de Desarrollos Regionales e Inca. El análisis de este contex to funerario consistió en el estudio de los restos óseos humanos, del ajuar funerario y de la urna en la que se efectuó el entierro. El hallazgo permitió avanzar en la comprensión de este tipo de prácticas mortuorias, inusuales en el área andina, asimismo fue posible plantear nuevas propuestas e interrogantes acerca de la ocupación tardía del Pucara de Perchel.

Palabras claves: Quebrada de Humahuaca, Período de Desarrollos Regionales, Pucara de Perchel, Entierro de Adultos en Urna.

\begin{abstract}
In this paper the finding of a primary adult urn burial in Pucara de Perchel is analyzed. Pucara de Perchel site has a surface of $2.921 \mathrm{~m}^{2}$ and it is located in Angosto del Perchel (Tilcara-Quebrada de Humahuaca) and its occupation was most important during the Regional Developments and Inca periods. The analysis of this funerary context consisted in the study of the human remains as well as grave goods and the burial urn. This finding allowed advancing in the understanding of this type of mortuary practice since the burial of adults in urns is unusual in the Andean area; also it was possible to state new hypothesis and questions about Pucara de Perchel late occupation.
\end{abstract}

Keywords: Quebrada de Humahuaca, Regional Development Period, Pucara de Perchel, Burial of Adults in Urns.

\section{Introducción}

El ritual mortuorio, en tanto rito de pasaje, marca una transición en la que interactúan el cuerpo y alma del difunto y también la sociedad a la que este pertenece. Desde esta perspectiva 
y como lo han señalado diversos autores (Bloch y Parry 1982; Parker Pearson 1993; Carr 1995; Charles y Buikstra 1995) el ritual funerario es realizado por los vivos y para los vivos, permitiendo que los muertos sean puestos al servicio de la creación y manipulación ideológica.

Si bien los rituales mortuorios en los Andes son variados y complejos, es recurrente la visión de la muerte como una parte de la vida en la que no se rompen los vínculos entre el difunto y la comunidad: el mundo de los muertos no es algo separado del de los vivos. De esta manera la experiencia de la muerte es tanto una experiencia personal como comunitaria (Aláez García 2001; Bascopé Caero 2001). Partiendo de estas consideraciones el presente trabajo tiene como objetivo analizar el entierro primario de un adulto en el interior de una urna, un contexto funerario rescatado en el Pucara de Perchel $^{1}$ (Scaro 2009). En base al objetivo propuesto, se plantea como hipótesis de trabajo que el contexto funerario en cuestión corresponde al período de Desarrollos Regionales (ca. 1.000 d.C.-1.430/80 d.C.) y da cuenta de vinculaciones entre el Pucara de Perchel, en el sector medio de la Quebrada de Humahuaca, y las Selvas Occidentales.

El entierro de adultos en urnas es una modalidad funeraria prácticamente inexistente en el área andina y son escasas las referencias acerca de su hallazgo. Las noticias más tempranas fueron presentadas por Lafone Quevedo (1891)², Ambrosetti (1906), Boman (1908), Weiser y Wolters (1920-1929), Torres (1921) y Debenedetti (1930) a finales del siglo XIX y principios del XX. Posteriormente Gatto (1946) y Berberián (1969) también realizaron hallazgos de este tipo. El descubrimiento más reciente de un entierro de adultos en urna corresponde al realizado por Williams y De Hoyos (2001).

El origen oriental de esta práctica funeraria fue señalado tempranamente. A principios del siglo XX se interpretaba la presencia de adultos enterrados en urnas en el área andina como una influencia de grupos Tupí-Guaraní del Brasil. Sin embargo, los hallazgos efectuados en La Candelaria (Salta) permitieron realizar nuevos planteos al respecto. Las diferencias observadas entre los materiales Candelaria y los de origen Guaraní brindaron elementos para rechazar la hipótesis de una "invasión" Guaraní en el Noroeste Argentino (Gatto 1946; Berberián 1969). Para explicar la presencia de esta práctica funeraria en el área Valliserrana, Berberián (1969) propuso que el entierro de adultos en urnas fue realizado desde momentos tempranos en las Selvas Occidentales del Noroeste. De acuerdo con el autor, en un determinado momento del período tardío esta práctica se habría difundido al área Valliserrana.

Williams y De Hoyos (2001) realizaron su hallazgo en el sitio Agua Verde, en la región de Andalgalá. Se trata del entierro múltiple en una urna de dos adultos y un subadulto acompañados por un ajuar cerámico conformado por vasijas de estilos locales e incaicos provinciales. Las autoras plantean que en el caso particular de Agua Verde se habrían inhumado a miembros de la élite relacionados con el Imperio Incaico, asentados en el cercano sitio Potrero-Chaquiago ( $3 \mathrm{~km}$ del entierro). Además, mencionan que en este sitio se han encontrado evidencias de colonos provenientes del piedemonte oriental, sector vinculado tradicionalmente con el origen de la costumbre de inhumación de adultos en urnas. 


\section{El Pucara de Perchel}

El Pucara de Perchel se sitúa en el departamento Tilcara (Figura 1), en el sector centralde la Quebrada de Humahuaca, un valle estrecho rodeado por altas cadenas montañosas que se extiende a lo largo de $120 \mathrm{~km}$ en la provincia de Jujuy. La misma se caracteriza por su gran heterogeneidad natural, generada por la combinación de su ubicación subtropical, una compleja geología, la existencia de una importante masa montañosa alineada en dirección norte-sur, y también por su marcada pendiente que permite la contigüidad de diversos entornos ambientales (Reboratti 2003).

La zona en la que se ubica Perchel corresponde a la Provincia Fitogeográfica de Prepuna (Cabrera 1976). La misma se caracteriza por una cobertura vegetal escasa de comunidades xerofíticas compuestas por cactáceas columnares (Trichocereus pasacana y Trichocereus tarijensis) y rastreras (Opuntia), y especies arbóreo-arbustivas espinosas, tales como el churqui (Prosopis feroz) (Braun Wilke et al. 2001).

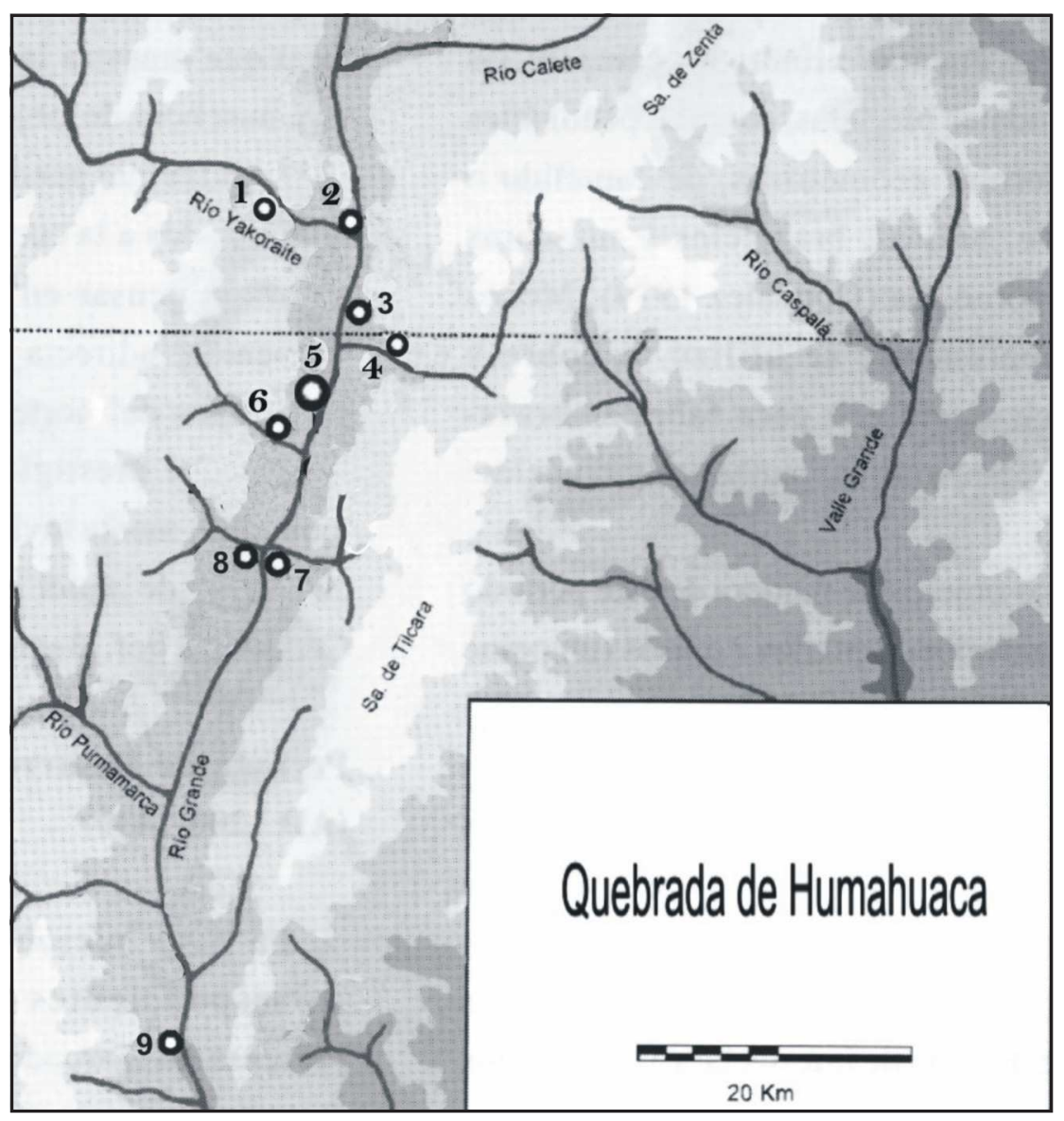

Figura 1. Ubicación de algunos sitios prehispánicos en la Quebrada de Humahuaca (Modificado de Nielsen 2001): 1. Los Amarillos. 2. Pucara de Yacoraite. 3. Campo Morado. 4. La Huerta. 5. Pucara de Perchel. 6. Pucara de Juella. 7. Pucara de Tilcara. 8. Pucara de Huichairas. 9. Pucara de Volcán. 
El sitio, de $2.921 \mathrm{~m}^{2}$ de superficie, se localiza a $2.700 \mathrm{msnm}$ en la parte más elevada de un morro en el Angosto de Perchel sobre la margen occidental del río Grande $\left(23^{\circ} 29^{\prime}\right.$ lat. sur $65^{\circ} 21^{\prime}$ long. oeste). Al estar ubicado sobre este angosto, es posible controlar visualmente el territorio comprendido entre Tilcara ( $11 \mathrm{~km}$ al sur del sitio) y Huacalera (6 km al norte). El angosto corresponde a un pedimento de erosión antiguo ${ }^{3}$ que tiene una altura de $100 \mathrm{~m}$ desde el fondo de valle. Las laderas de mismo caen abruptamente hacia el norte y hacia el este. Estas abruptas laderas hacen muy difícil el acceso al Pucara por estos sectores, quedando éste restringido a la ladera sur.

La ubicación estratégica del Pucara de Perchel en lo alto de un angosto, el difícil acceso al sitio y el dominio visual de una gran distancia permiten plantear que la función de Perchel se vincularía con la defensa de territorios y el control de redes de intercambio.

Las construcciones relevadas en el sitio se disponen en tres escalones altitudinales. El más alto está en la cima del cerro (a $2.709 \mathrm{msnm}$ ), en este sector la estructura predominante es una pequeña plataforma circular arqueológica sobre la que se construyó una apacheta moderna. El segundo escalón altitudinal está en el espolón sur, localizado en sentido transversal al fondo de valle, a $2.698 \mathrm{msnm}$. En este sector se ubica la menor cantidad de estructuras arqueológicas aunque su conservación es muy buena. Además, allí se ubica el acceso al sitio. El tercer escalón se ubica en el espolón norte, a $2.690 \mathrm{msnm}$. Es aquí donde se observa la mayor densidad de estructuras arqueológicas, aunque su conservación es muy pobre debido al importante declive de las laderas.

Los recintos observados en superficie presentan diferencias a nivel arquitectónico, siendo posible distinguir dos tipos de estructuras (Figura 2). El primero (Figura 2a) incluye recintos rectangulares con muros dobles conformados por bloques de cuarcita de color rosado a morado (materia prima que aflora en la ladera norte del sitio), los bloques están bien canteados y son de tamaños regulares. Estas estructuras están asociadas a una hornacina, a escalinatas de piedra y a "torreones".

El segundo tipo de estructuras (Figura 2b) presenta muros dobles o simples conformados con bloques de diversas materias primas (cuarcita, filita y arenisca), que tienen tamaños
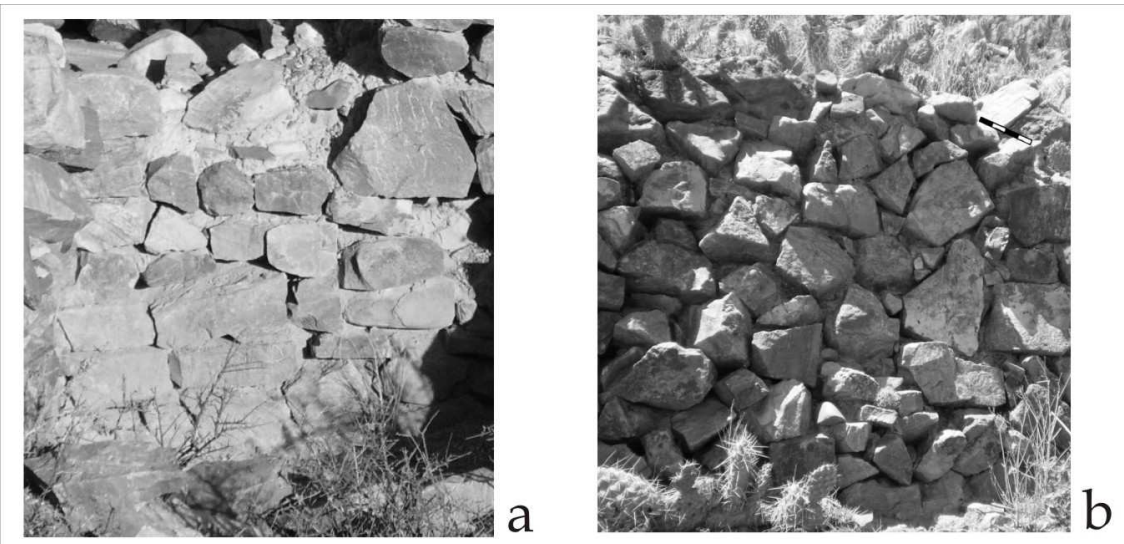

Figura 2. Arquitectura del Pucara de Perchel. a. Estructuras de bloques bien canteados. b. Lienzo poco cuidado. 
diferentes y sus superficies están poco canteadas o son naturalmente aplanadas. Estas características dan al lienzo un aspecto irregular.

A partir de las diferencias observadas a nivel arquitectónico se planteó como hipótesis de trabajo la presencia de dos momentos en la construcción de las estructuras del Pucara de Perchel. El momento más temprano, evidenciado en el segundo tipo de estructura, correspondería por lo menos al período de Desarrollos Regionales (ca. 1.000 d.C.-1.430/80 d.C.), mientras que el primer tipo de estructuras corresponderían a la dominación incaica de la región.

\section{El rescate de un contexto funerario}

En abril de 2008 se rescató una urna funeraria descubierta por un aficionado a la arqueología luego de las últimas lluvias de verano. Esta persona excavó parcialmente el hallazgo hasta encontrar restos humanos, decidiendo denunciarlo. El contexto se localizaba en el espolón norte (Figura 3) y correspondía a una gran urna en la que se había realizado el entierro de un individuo adulto.

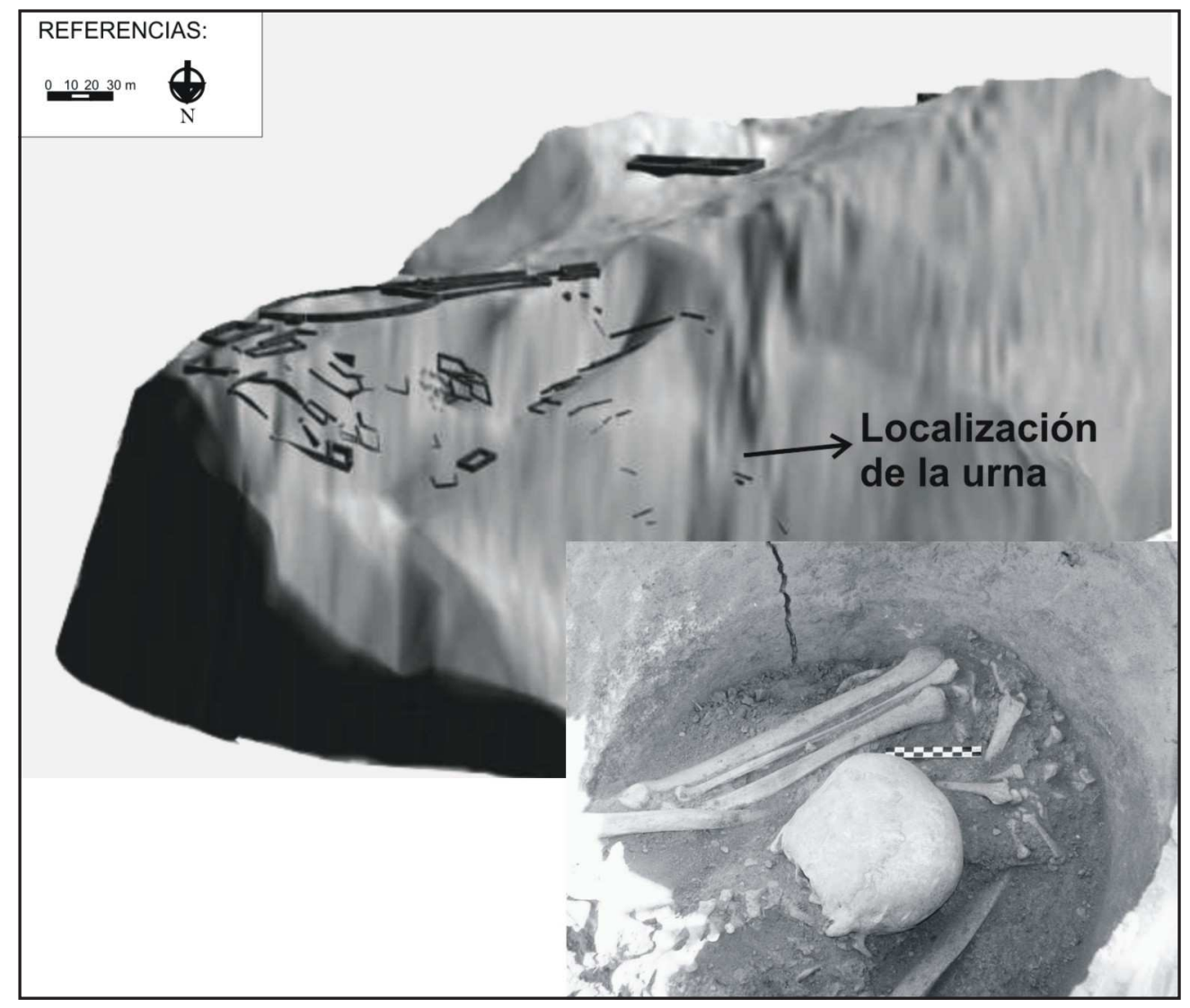

Figura 3. Vista del Pucara de Perchel desde el norte. Se indica el lugar donde fue encontrado el contexto funerario. 
Al retomar la excavación, se observaron fragmentos cerámicos de diversos tamaños sin ningún orden, alterados por quien realizara el hallazgo. Algunos de estos fragmentos correspondían a la urna en la que fue realizada la inhumación mientras que otros pertenecían al ajuar funerario. El individuo enterrado fue hallado a $25 \mathrm{~cm}$ de la superficie, dentro de la urna y con el cráneo orientado hacia el sur. El esqueleto (Figura 3) estaba completamente articulado, indicando que se trataba de un entierro primario, en posición genuflexa con las manos cruzadas sobre las rodillas y la cabeza apoyada sobre los brazos.

Una vez retirado el cuerpo se encontraron fragmentos de laja de color verde y morado y de cuarcita rosada que hacían las veces de base de la gran urna. Por debajo de esta se halló una lasca de obsidiana pequeña. Alrededor de la urna se observó un sedimento arcilloso, indicando tal vez la preparación del lugar antes del entierro.

El carbón hallado entre los huesos del individuo fue fechado, arrojando un resultado de 737 \pm 29 A.P. (carbón-AA88374, d13C=-23.3\%o-AMS). Calibrado este fechado resultó en 1.2601.285 Cal. D.C. (1 sigma) y 1.225-1.291 Cal. D.C. (2 sigmas) (Cal. con el programa OxCal 4.1. [Ramsey 2010]).

\section{La urna}

La urna (Figura 4) en la que estaba enterrado el individuo corresponde a una vasija cerrada de forma subglobular con asas en cinta ubicadas en el cuerpo superior. Esta vasija de gran tamaño tiene un diámetro máximo de $56 \mathrm{~cm}$. La superficie externa de la vasija presenta una gruesa capa de hollín mientras que su interior es ordinario de color rojizo. La pasta corresponde al estándar 16 de los estándares de pasta propuestos por Cremonte (1991) para la Quebrada de Humahuaca, el mismo se caracteriza por ser bastante compacta con inclusiones en su mayoría marrones y en menor proporción grises (rocas sedimentarias y metamórficas). Los tamaños de las inclusiones no son uniformes aunque destacan las medianas y algunas grandes equidimensionales subredondeadas. Las cavidades presentes son abundantes y sin orden.

La urna exhibe el cuello y la base intencionalmente seccionados, probablemente con el fin de introducir el cuerpo. Esta práctica es recurrente en los sitios de la Quebrada, observándose en las urnas usadas para el entierro de párvulos halladas en el Pucara de Volcán y en el Pucara de Tilcara. El entierro en vasijas cerámicas cortadas intencionalmente da cuenta de la reutilización de estas piezas que tenían en un principio otra función. Gatto (1946) señaló

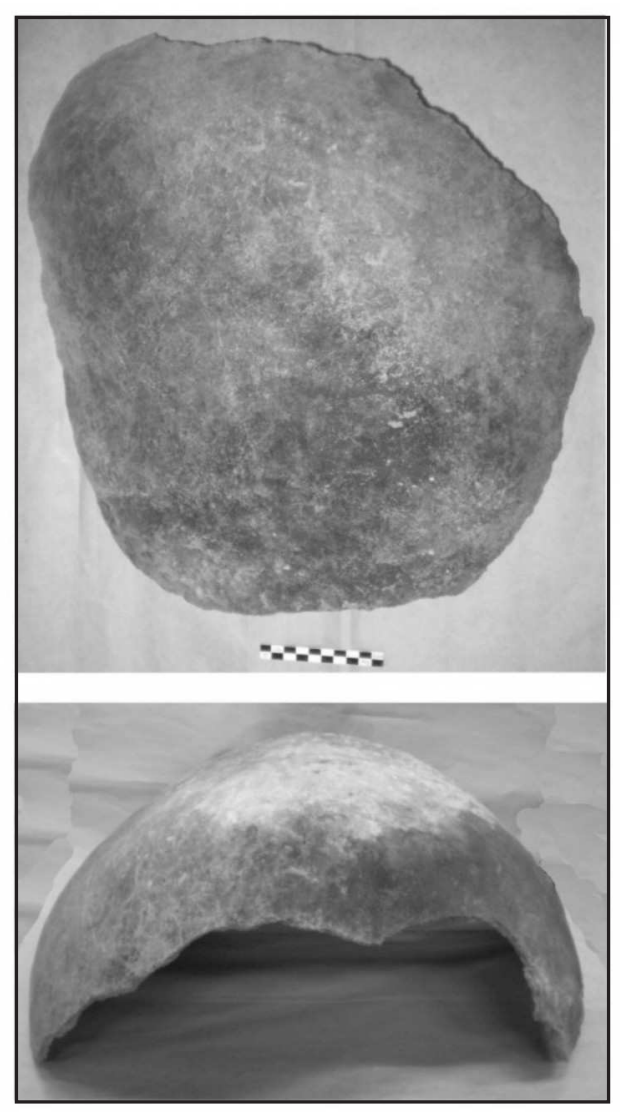

Figura 4. La urna funeraria. 
que en Volcán algunas de las urnas en las que fueron enterrados párvulos presentan manchas de hollín en el exterior, delatando un "uso común" previo.

\section{Análisis de los restos óseos}

El análisis osteológico del individuo enterrado (Figura 5) indica que se trataba de un hombre con una edad estimada en $32 \pm 4$ años al momento de su muerte. El sexo se determinó a través de la observación de variables morfológicas del cráneo (cresta nucal, proceso mastoideo, margen supraorbital, glabela y eminencia mentoniana) y del coxal (arco ventral, concavidad subpúbica, rama isquiopúbica, escotadura ciática mayor y surco preauricular) según los estándares de Buikstra y Ubelaker (1994).

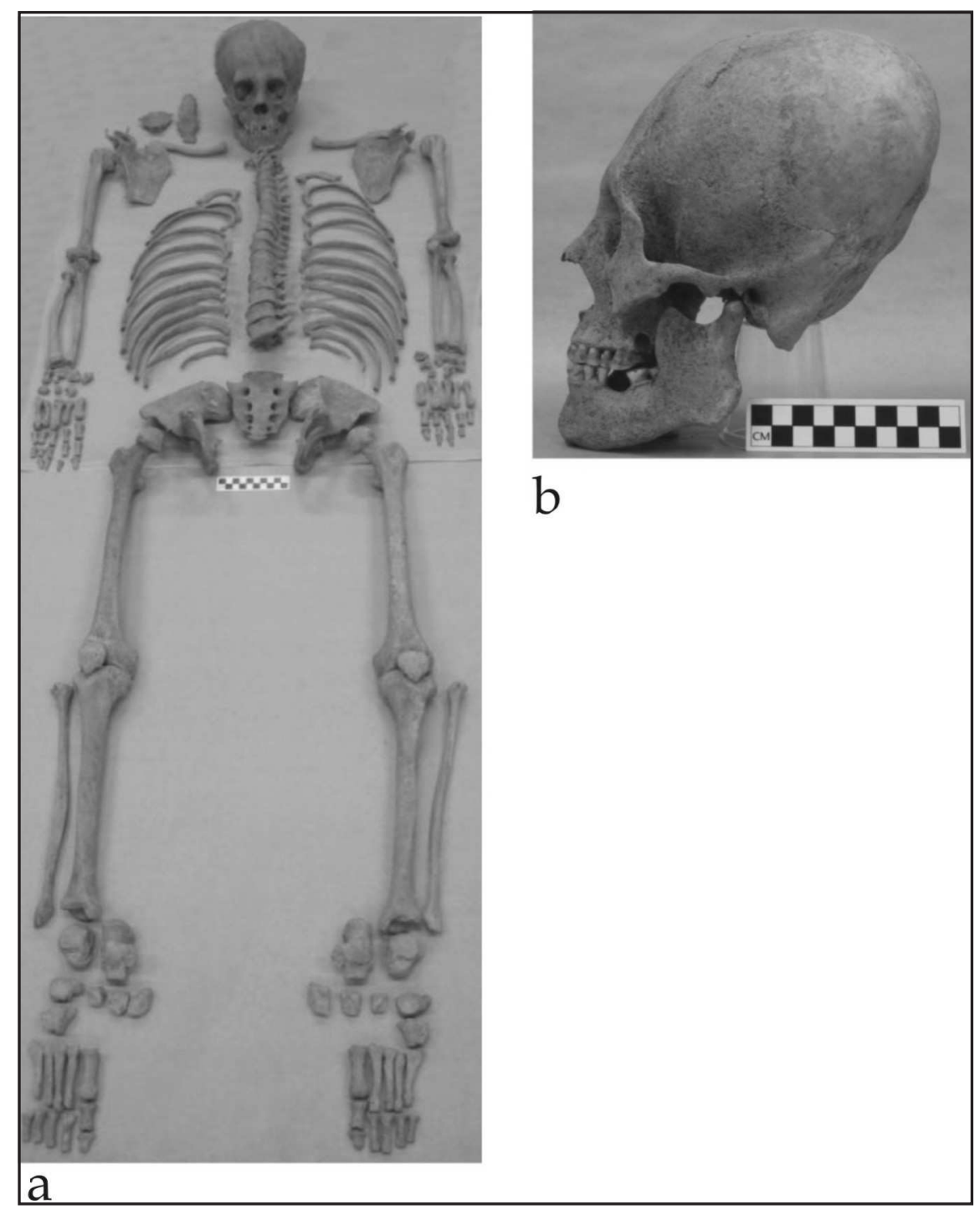

Figura 5. a. El individuo inhumado en el interior de la urna. b. Deformación craneana del tipo tabular oblicua (vista lateral izquierda del cráneo). 
La edad de muerte se estimó de acuerdo a la secuencia de obliteración de las suturas maxilares (Mann et al. 1987), los cambios morfológicos de la sínfisis púbica, utilizando tanto el método de Todd (1921) como el de Suchey- Brooks (Brooks y Suchey 1990) y la superficie auricular del ilion, siguiendo a Lovejoy et al. (1985).

Este adulto joven (Figura 5a) habría tenido una estatura en vida de $159 \pm 2,81 \mathrm{~cm}$ según fue estimado a partir de la longitud máxima de la tibia y siguiendo las formulas propuestas por Genovés (1967)4. El cráneo muestra una deformación de tipo tabular oblicua (Figura 5b) muy simétrica y proporcionada (Gheggi 2009).

No se observan lesiones traumáticas o patologías infecciosas o nutricionales. Por otra parte, sus piezas dentales están bien conservadas y sólo presentan un desgate leve sin advertirse caries o cálculos dentales. Se registró la pérdida de piezas dentales antemortem (los dos primeros molares del maxilar) y la presencia de un absceso en el segundo molar superior izquierdo (Ortner y Putschar 1981; Aufderheide y Rodríguez-Martín 1998; Langsjoen 1998).

Se observaron lesiones de tipo degenerativo en el sector lumbar de la columna vertebral y en la articulación coxo-femoral. La primera lesión corresponde a la presencia de un proceso de destrucción ósea (proceso osteolítico) y de generación ósea (osteofitosis) de la quinta vértebra lumbar que coincide a su vez con un proceso proliferativo óseo en forma de estrella sobre la superficie superior del cuerpo de la primera vértebra sacra. Además, sobre la superficie articular izquierda del sacro se observa depositación ósea, la cual coincide con un proceso osteolítico presente sobre la superficie auricular del coxal izquierdo (Ortner y Putschar 1981; Barnes 1994; Aufderheide y Rodríguez-Martín 1998).

Fue posible determinar que las mencionadas lesiones son coincidentes con una patología articular degenerativa producto de un continuo y exigido uso de las articulaciones de la columna a nivel lumbar y de la cadera. Si bien el proceso degenerativo de las articulaciones es inherente al avance de la edad al producirse el desgaste de los cartílagos interarticulares, el uso continuo y exigido de ciertas regiones articulares las expone a un mayor riesgo. En el caso que nos ocupa, las lesiones presentes indican que el individuo desarrolló una actividad física cotidiana que involucraba especialmente ambos miembros inferiores y la cintura.

\section{El ajuar}

Como fue mencionado, el ajuar estaba ubicado por sobre el esqueleto del individuo inhumado y se componía de 37 fragmentos cerámicos sin orden. Los fragmentos corresponden a un número mínimo ${ }^{5}$ de 5 vasijas (Figura 6), asignables por sus características estilísticas y de las de sus pastas a la alfarería típica de la Quebrada de Humahuaca.

A una primera vasija (Figura 6a) corresponden 5 fragmentos; es una vasija ordinaria cerrada de forma no determinable cuyo diámetro máximo es de $36 \mathrm{~cm}$. La superficie de la misma es de color castaño y presenta restos de hollín en el cuerpo inferior. Las características de la pasta de esta vasija permiten incluirla en el estándar 22 de los estándares propuestos por Cremonte (1991) para la Quebrada de Humahuaca. Se trata de una pasta poco compacta con inclusiones de rocas grises, moradas y amarillentas de tamaño mediano a muy grande. 


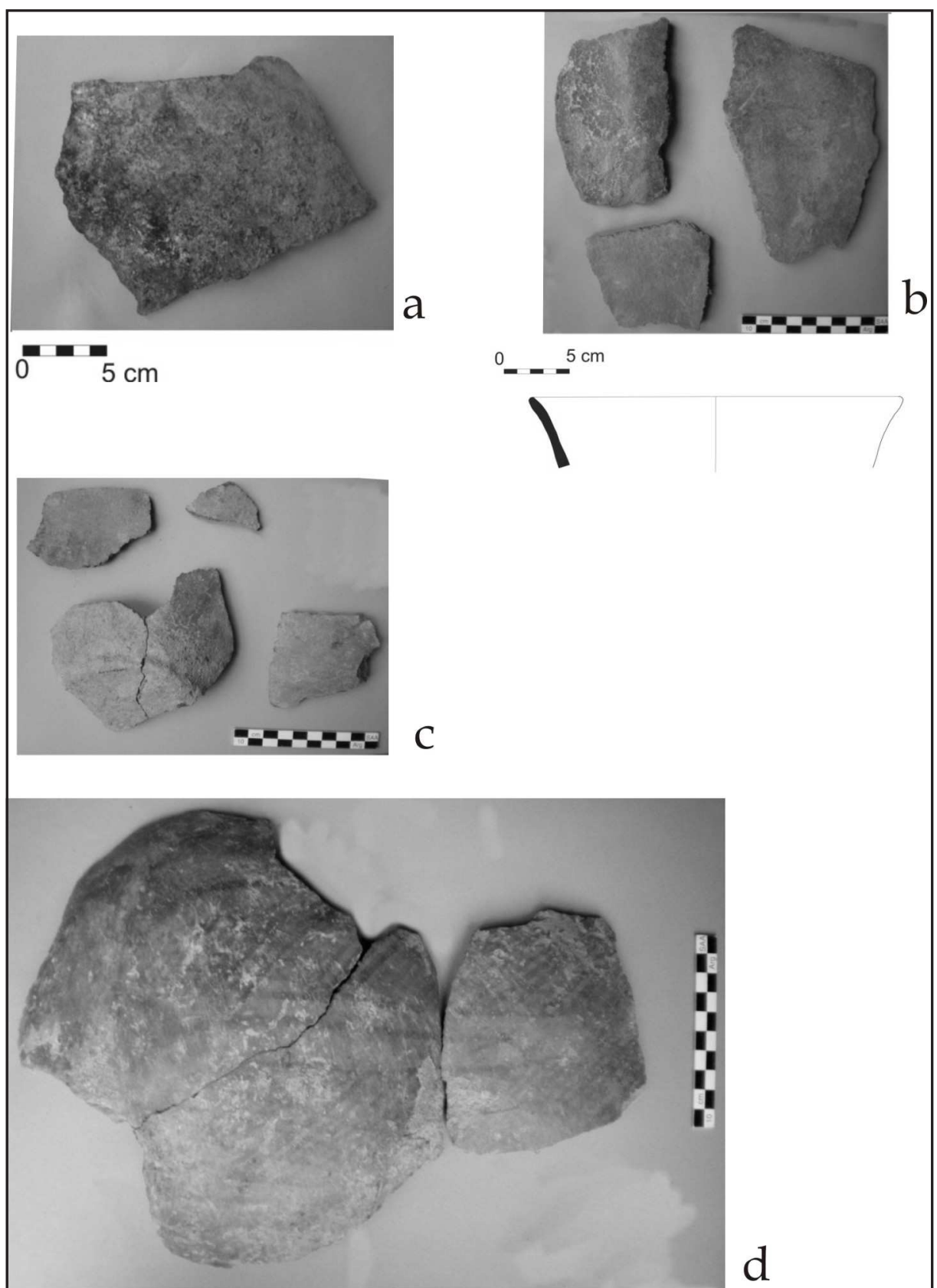

Figura 6. Fragmentos de las vasijas del ajuar funerario. a. Vasija 1. b. Vasija 2. c. Vasija 3.

$$
\text { d. Vasija } 4 .
$$

Estas inclusiones son de forma tabular y también equidimensional subredondeada. Las cavidades no son muy abundantes y no presenta ningún orden.

La segunda vasija (Figura 6b) está representada por 13 fragmentos de un cántaro ordinario negro de $30 \mathrm{~cm}$ de diámetro de abertura y de paredes bastante gruesas ( 8 a $13 \mathrm{~mm}$ ). Su superficie externa presenta huellas de hollín, al igual que la vasija 1. La pasta de esta vasija 
corresponde al estándar 21 de los propuestas para la Quebrada de Humahuaca por Cremonte (1991), y se caracteriza por ser compacta, con inclusiones de fragmentos líticos marrones en general de tamaño no uniforme, aunque se destacan la grandes. Las inclusiones pueden tener forma tabular o equidimensional subredondeada.

Se encontraron 10 fragmentos pertenecientes a una tercer vasija (Figura 6c). La misma es cerrada de forma no determinable con una base plano convexa de $28 \mathrm{~cm}$ de diámetro y paredes de $6 \mathrm{~mm}$ de espesor. La superficie externa de esta vasija es pulida de color no uniforme que varía entre el rojo y el morado (7.5R $5 / 4$ y 7.5R 2.5/4), su superficie interna es ordinaria rojiza. Esta vasija corresponde al estándar 4 (Cremonte 1991) de acuerdo a las características de su pasta, la que es bastante compacta y presenta inclusiones de rocas marrones y escasas grises y blancas. Las inclusiones son de tamaño no uniforme, aunque las medianas y grandes son más abundantes. Las cavidades no son muy abundantes y son en general alargadas.

La vasija 4 (Figura 6d) se está representada por 8 fragmentos, es cerrada, su diámetro máximo es de $40 \mathrm{~cm}$ y sus paredes tienen un espesor de $6 \mathrm{~mm}$. La superficie externa es pulida y está decorada con diseños pintados en negro sobre fondo rojo (10R 4/3 y 2.5 YR 4/4); los mismos corresponden a arriñonados reticulados en línea fina asociados a líneas negras finas que terminan en una flecha. La pasta de la vasija corresponde al estándar 24 (Cremonte 1991) y se caracteriza por ser compacta con inclusiones de fragmentos de rocas marrones, grises y blancas. Las inclusiones marrones y grises son en su mayoría de tamaño mediano y grande, de forma tabular, mientras que las blancas son de tamaño pequeño y de forma equidimensional redondeada. Las cavidades observadas son abundantes, grandes y no tienen orden.

El ajuar se completa con un fragmento aislado, correspondiente a una quinta vasija de forma no determinable cuya superficie externa es roja pulida lisa.

La presencia de la vasija decorada en negro sobre rojo permite plantear que este contexto corresponde a momentos tardíos, ya que el diseño de las "flechas" aparece durante el período de Desarrollos Regionales y continúa durante el Incaico (Nielsen 1997). En principio se pensó que el contexto funerario correspondía al momento incaico por la presencian de esta vasija, ya que el diseño presenta línea fina y la superficie está muy pulida. Asimismo el color de fondo, muy irregular en principio se consideró como castaño, aunque posteriormente se pudo constatar que correspondía a tonos de rojo. La línea fina y la superficie pulida aumentan significativamente durante el Incaico de acuerdo a lo constatado por Cremonte (com. pers. 2009) en el Pucara de Volcán y Esquina de Huajra, sitios ubicados en el sector centro-sur de la Quebrada.

\section{Discusión}

El contexto funerario analizado en este trabajo se suma a los escasos ejemplos de inhumaciones de adultos en urnas de la Quebrada de Humahuaca. Estos hallazgos fueron realizados a principio del siglo XX en los sitios Pucara de Volcán, Pucara de Tilcara y La Huerta. En el Pucara de Volcán, Gatto (1946) encontró un adulto sepultado en el interior de una gran urna en el fondo de la denominada quebrada de la Mina. El autor la describe como una urna ordinaria aunque de buena cocción, de forma subglobular con cuello amplio y 
evertido y base circular plana. Los restos óseos correspondían a un individuo adulto del que sólo se rescataron el cráneo y los huesos largos, ya que los elementos más pequeños del esqueleto estaban en malas condiciones de conservación. Gatto no especifica si este entierro estaba acompañado por algún ajuar funerario.

En el Pucara de Tilcara, Debenedetti (1930) encontró un adulto enterrado en una vasija cerámica en una cámara en el ángulo noroeste de un edificio rectangular (yacimiento 149). Este enterratorio fue realizado en el interior de un virque que estaba seccionado en la parte superior con el fin de introducir al individuo enterrado sin ajuar. Los restos óseos de este entierro estaban totalmente destruidos. El hallazgo de La Huerta también fue realizado por Debenedetti quien propone que se trataría de un "probable chiriguano adulto" (Raffino 1993: 92).

En relación con los entierros de adultos en urna localizados en la Quebrada, el contexto funerario rescatado en el Pucara de Perchel permite contar con datos concretos sobre cronología y sobre las características bioarqueológicas del individuo inhumado, ya que en relación con los otros casos de la zona, el de Perchel se destaca por la excelente conservación de los restos óseos.

Por otra parte, el hallazgo de un adulto inhumado en una urna en Perchel contribuyó a la modificación de la visión que se tenía acerca del área residencial del sitio durante el período de Desarrollos Regionales. En un principio se consideraba que el sector en el que fue recuperada la urna era un área marginal del sitio en la que sólo existirían muros de contención. Sin embargo, el contexto rescatado permitió plantear que el sector residencial sería de mayor tamaño y complejidad de lo que se había supuesto inicialmente.

Por último, la presencia en Perchel de una práctica mortuoria prácticamente inexistente en las tierras altas podría indicar la vinculación del sitio con poblaciones provenientes del oriente, de la zona de las Selvas Occidentales, por lo menos durante el período de Desarrollos Regionales. La misma incluiría desplazamientos de individuos y a la vez integración cultural de los mismos, si consideramos las características propias de la Quebrada de las vasijas que conformaban el ajuar funerario. Además, el caso presentado es el primer contexto de la Quebrada de Humahuaca de un adulto enterrado en urna que cuenta con una datación radiocarbónica que refleja la existencia de esta práctica funeraria en momentos pre-incaicos.

\section{Agradecimientos}

Agradecemos a Nicolás Lamberti, Daniel Ochoa y Sebastián Peralta la colaboración en las tareas de campo y gabinete. A Gabriel Lamas en la elaboración de gráficos en 3D y a las geólogas Natalia Solís y Alba Ramírez.

\section{Notas}

${ }^{1}$ Este trabajo forma parte de la investigación realizada en el marco de la Tesis de Licenciatura de la Facultad de Humanidades y Ciencias Sociales, Universidad Nacional de Jujuy.

${ }^{2}$ La colección de Chañar Yaco recuperada por Lafone Quevedo fue recientemente revisada por Moralejo (2010).

${ }^{3}$ Un pedimento de erosión corresponde a una zona de transición entre áreas elevadas y las más bajas que forma parte del relieve poligénico (Solís y Ramírez 2008: 440). 
${ }^{4}$ La estatura viva fue estimada a partir de la longitud máxima de la tibia según la fórmula presentada por Genovés (1967) sobre restos cadavéricos de indígenas mesoamericanos (estatura viva $=1,96^{*}$ (tibia)+93,75+/-2,81). La misma presenta un desvío estándar menor que la fórmula del fémur del mismo autor.

${ }^{5}$ El Número Mínimo de Vasijas se calculó asignando fragmentos a un mismo recipiente. El mismo se realizó teniendo en cuenta el tratamiento y acabado de las superficies, las características macroscópicas de la pasta y el grosor de los fragmentos.

\section{Bibliografía citada}

Aláez García, A.

2001 Duelo andino: sabiduría y elaboración de la muerte en los rituales mortuorios. Chungara 33 (2): 173-178.

Ambrosetti, J.B.

1906 Exploraciones arqueológicas en la Pampa Grande (provincia de Salta). Publicaciones de la sección antropológica 1.

Aufderheide, A. y C. Rodríguez-Martín

1998. The Cambridge Encyclopedia of Human Paleopathology. Cambridge University Press, Cambridge.

Barnes, E.

1994. Developmental defects of the axial skeleton in paleopathology. University Press of Colorado. Niwot, Colorado.

Bascopé Caero, V.

2001 El sentido de la muerte en la cosmovisión andina; el caso de los valles andinos de Cochabamba. Chungara 33 (2); 271-277.

Berberián, E.

1969 Entierros de adultos en urnas en el área Valliserrana del NOA. Revista del Instituto de Antropología 29: 3-71.

Boman, E.

1908 Antiquitiés de la région andine de la République Argentine et du désert d'Atacama. Ediunju, Jujuy.

Bloch, M. y J. Parry.

1982 Death and the regeneration of life. Cambridge University Press, Cambridge.

Braun Wilke, R., E. Santos, L. Picchetti, M. Larran, G. Guzman, C. Colarich y C. Casoli 2001 Carta de aptitud ambiental de la Provincia de Jujuy. Ediunju, Jujuy.

Brooks, S.T. y J.M. Suchey.

1990. Skeletal age determination based on the os pubis: a comparison of the Acsadi-Nemeskeri and Suchey-Brooks methods. Human Evolution 5: 227-238. 
Buikstra, J.E. y D.H. Ubelaker

1994. Standards for data collection from human skeletal remains. Arkansas Archaeological Survey research Series No. 44. Fayetteville, Arkansas.

Cabrera, A.L.

1976 Regiones Fitogeográficas Argentinas. Enciclopedia argentina de agricultura y jardinería, Tomo 2, Fascículo 1 (ed. por Parodi y Dimitri), pp. 1-85. ACME, Buenos Aires.

Carr, C.

1995 Mortuary Practices: their Social, Philosophical - Religious Circumstantial, and Physical Determinants. Journal of Archaeological Method and Theory 2 (2):105-200.

Charles, D. y J. Buikstra.

1995 Structural evidence of ritual practice at the Hopewell Mound House site in west-central Illinois. Society for American Archaeology. Minneapolis Minnesota.

Cremonte, M.B.

1991 Análisis de muestras cerámicas de la Quebrada de Humahuaca. Avances en Arqueología 1: 7-42.

Debenedetti, S.

1930 Las ruinas del pucará. Tilcara, Quebrada de Humahuaca (Provincia de Jujuy). Archivos del Museo Etnográfico 11.

Gatto, S.

1946 Exploraciones arqueológicas en el Pucara de Volcán. Revista del Museo de La Plata 6: 691.

Genovés, S.

1967. Proportionality of the Long Bones and their Relation to Stature among Mesoamericans. American Journal of Physical Anthropology 26: 67-78.

Gheggi, M. S.

2009 Informe sobre el análisis de los restos óseos humanos procedentes del Pucará de Perchel. Buenos Aires. MS

Lafone Quevedo, S. A.

1891 Las Huacas de Chañar Yaco (Provincia de Catamarca). Revista del Museo de La Plata 2: 353-360

Langsjoen, $\mathrm{O}$.

1998 Diseases of the dentition. The Cambridge Encyclopedia of Human Paleopathology (editado por A. Aufderheide y C. Rodríguez-Martín), pp. 393-412. Cambridge University Press. Cambridge.

Lovejoy, C.O., R.S. Meindl, T.R. Pryzbeck y R.P. Mensforth 1985 Chronological Metamorphosis of the Auricular Surface of the Ilium: a new method for the determination of age at death. American Journal of Physical Anthropology 63: 15-28. 
Mann R.W., Symes S.A. y W.M. Bass

1987 Maxillary suture obliteration: aging the human skeleton based on intact or fragmentary maxilla. Journal of Forensic Science 32:148-157

Moralejo, R. A.

2010 Las Huacas de Chañar Yaco: importancia de su significado y su revisión. Revista Española de Antropología Americana 40 (2): 51-76.

Nielsen, A. E.

1997 Tiempo y cultura material en la quebrada de Humahuaca. 700-1650 d.C. Instituto Interdisciplinario Tilcara (UBA). Jujuy.

Ortner, D. y W. Putschar

1981 Identification of Pathological Conditions in Human Skeletal Remains. Smithsonian Institution Press, Washington.

Parker Pearson, $\mathrm{M}$.

1993 The powerful dead: archaeological relationships between the living and the dead. Cambridge Archaeological Journal 3: 203-229.

Raffino, R. A.

1993 INKA. Arqueología, historia y urbanismo del Altiplano Andino. Corregidor, Buenos Aires.

Ramsey, C.

2010 Oxcal 4.1. Oxford University. http:/ /c14.arch.ox.ac.uk/

Reboratti, C.

2003 La Quebrada. La Colmena, Buenos Aires.

Scaro, A.

2009 El Pukara de Perchel (Til 4). Arqueología e Historia de un lugar estratégico en la Quebrada de Humahuaca (Jujuy, Argentina). Tesis de Licenciatura, Facultad de Humanidades y Ciencias Sociales, Universidad Nacional de Jujuy, Jujuy.

Solís, N. y A. Ramírez

2008 Geomorfología de la Provincia de Jujuy. Geología y recursos naturales de la Provincia de Jujuy (ed. por B. Coira y E. Zapettini), pp. 433-442. Ediunju, Jujuy.

Torres, L.

1921 Arqueología de los Valles orientales de la Pcia. de Salta. Urnas funerarias en la cuenca del Rio Rosario (Dto. De Rosario de la Frontera). Revista del Museo de La Plata (S. A.) 15: 1-14.

Weiser, V y F. Wolters.

1920-1929. Diarios y libretas de campo de las expediciones Benjamín Muniz Barreto. Departamento de Arqueología. Museo de La Plata.

Williams, V. y M. de Hoyos.

2001. El entierro de Agua Verde. Variables bioarqueológicas para el estudio de la complejización social. Intersecciones en Antropología 2: 19-34. 